\title{
CORRIGENDUM
}

\section{A systematic review: antipsychotic augmentation with treatment refractory obsessive-compulsive disorder}

\author{
MH Bloch, A Landeros-Weisenberger, B Kelmendi, V Coric, MB Bracken and JF Leckman
}

Molecular Psychiatry (2006) 11, 795. doi:10.1038/sj.mp.4001859

Correction to: Molecular Psychiatry (2006) 11, 622-632.

doi: $10.1038 /$ sj.mp.4001823

Following publication of the above article, the authors noted the following: while the captions for
Figures 4 and 5 were correct, the actual figures themselves were incorrectly transposed. They appear correctly below:

\begin{tabular}{|c|c|c|c|c|c|}
\hline $\begin{array}{l}\text { Study } \\
\text { or sub-category }\end{array}$ & $\begin{array}{l}\text { Antipsychotic } \\
n / N\end{array}$ & $\begin{array}{l}\text { Placebo } \\
\mathrm{n} / \mathrm{N}\end{array}$ & $\begin{array}{c}\mathrm{RD} \text { (fixed) } \\
95 \% \mathrm{Cl}\end{array}$ & $\begin{array}{c}\text { Weight } \\
\%\end{array}$ & $\begin{array}{c}\mathrm{RD} \text { (fixed) } \\
95 \% \mathrm{Cl}\end{array}$ \\
\hline \multicolumn{6}{|c|}{01 Maximal Dose SRI Therapy for less than 12 weeks } \\
\hline Shapira 2004 & $5 / 22$ & $4 / 22$ & $\rightarrow-$ & 15.91 & $0.05[-0.19,0.28]$ \\
\hline Carey 2005 & $8 / 20$ & $7 / 21$ & & 14.82 & $0.07[-0.23,0.36]$ \\
\hline Subtotal $(95 \% \mathrm{Cl})$ & & 43 & & 30.73 & $0.06[-0.13,0.24]$ \\
\hline \multicolumn{6}{|c|}{ Total events: 13 (Antipsychotic), 11 (Placebo) } \\
\hline \multirow{2}{*}{\multicolumn{6}{|c|}{$\begin{array}{l}\text { Test for heterogeneity: } \mathrm{Chi}^{2}=0.01, \mathrm{df}=1(\mathrm{P}=0.91), \mathrm{I}^{2}=0 \% \\
\text { Test for overall effect: } Z=0.58(P=0.56)\end{array}$}} \\
\hline & & & & & \\
\hline \multicolumn{6}{|c|}{02 Maximal Dose SRI Monotherapyfor at least 12 weeks } \\
\hline McDougle1994 & $5 / 17$ & $0 / 17$ & & 12.30 & $0.29[0.07,0.52]$ \\
\hline McDougle2000 & 7/20 & $0 / 16$ & & 12.86 & $0.35[0.13,0.57]$ \\
\hline Hollander 2003 & $3 / 10$ & $0 / 6$ & & 5.43 & $0.30[-0.03,0.63]$ \\
\hline Bystritsky2004 & 4/13 & $0 / 13$ & 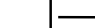 & 9.40 & $0.31[0.04,0.57]$ \\
\hline Denys 2004 & $8 / 20$ & $2 / 20$ & — & 14.47 & $0.30[0.05,0.55]$ \\
\hline Erzegovesi2005 & $5 / 10$ & $2 / 10$ & + & 7.23 & $0.30[-0.10,0.70]$ \\
\hline Fineberg 2005 & $1 / 11$ & $0 / 10$ & $t=-$ & 7.58 & $0.09[-0.13,0.31]$ \\
\hline Subtotal $(95 \% \mathrm{Cl})$ & 101 & 92 & & 69.27 & $0.29[0.18,0.39]$ \\
\hline \multicolumn{6}{|c|}{ Total events: 33 (Antipsychotic), 4 (Placebo) } \\
\hline \multirow{2}{*}{\multicolumn{6}{|c|}{$\begin{array}{l}\text { Test for heterogeneity: } \mathrm{Chi}^{2}=3.30, \mathrm{df}=6(\mathrm{P}=0.77), \mathrm{I}^{2}=0 \% \\
\text { Test for overall effect: } Z=5.29(P<0.00001)\end{array}$}} \\
\hline & & & & & \\
\hline & 143 & 135 & & 100.00 & $0.22[0.12,0.31]$ \\
\hline \multicolumn{6}{|c|}{ Total events: 46 (Antipsychotic), 15 (Placebo) } \\
\hline \multicolumn{6}{|c|}{ Test for heterogeneity: $\mathrm{Chi}^{2}=7.35, \mathrm{df}=8(\mathrm{P}=0.50), \mathrm{I}^{2}=0 \%$} \\
\hline Test for overall eff & $0.00001)$ & & & & \\
\hline
\end{tabular}

Figure 4

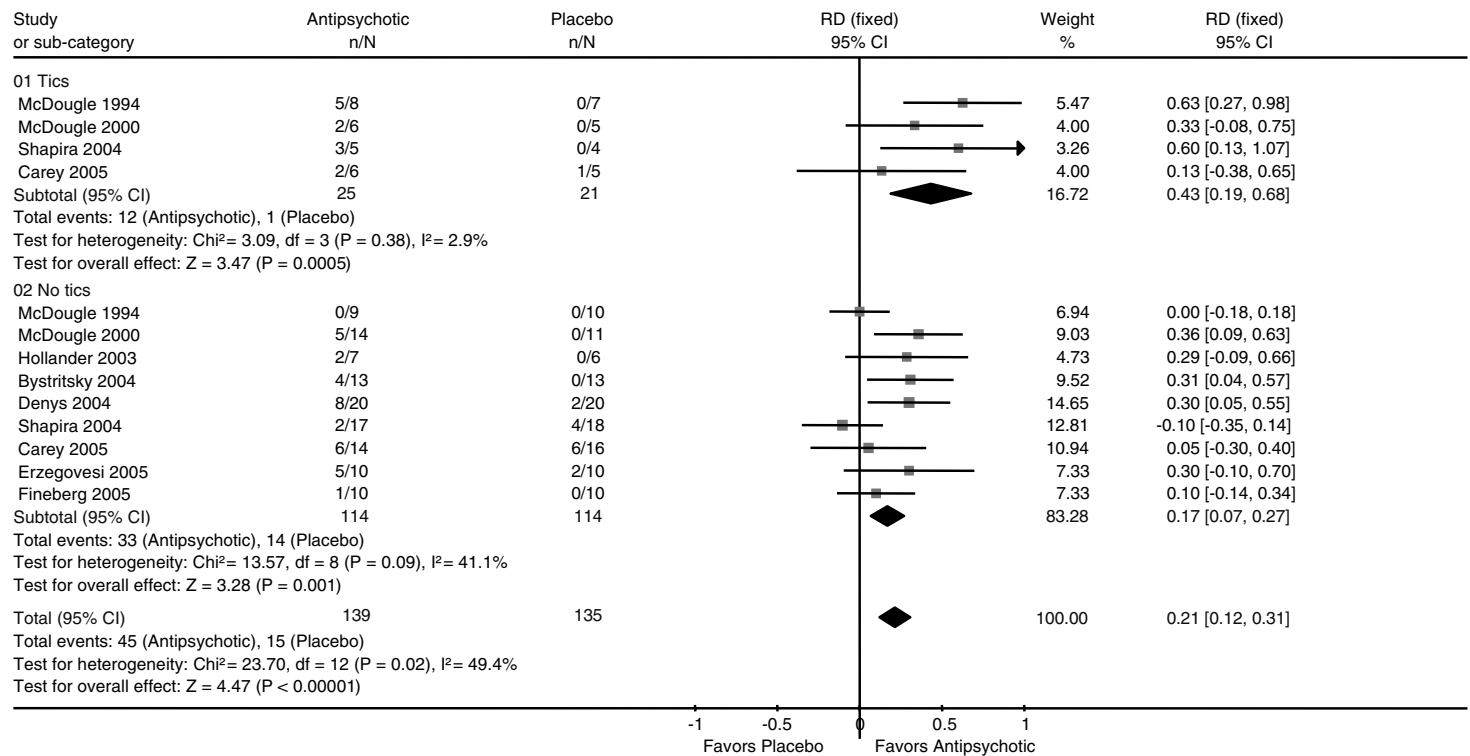

Figure 5 